\title{
Femtosecond laser triggering of a sub-100 picosecond jitter high-voltage spark gap
}

\author{
B. M. Luther, ${ }^{\text {a) }}$ L. Furfaro, A. Klix, and J. J. Rocca \\ Department of Electrical and Computer Engineering, Colorado State University, Fort Collins, \\ Colorado 80523
}

(Received 13 August 2001; accepted for publication 10 September 2001)

\begin{abstract}
We have demonstrated sub-100 ps jitter operation of a pressurized high-voltage air spark gap triggered by a femtosecond Ti:Sapphire laser. Time delay statistical fluctuations with a standard deviation as low as $+-\sigma=0.037 \mathrm{~ns}$ were obtained. (C) 2001 American Institute of Physics.
\end{abstract}

[DOI: $10.1063 / 1.1419036]$

Applications in several fields require either accurate triggering of high-voltage spark gaps capable of switching large currents, or very good synchronization between laser pulses and events controlled by these high-voltage switches. This has motivated a significant amount of research in lasertriggered spark gaps utilizing a variety of laser sources and triggering mechanisms. Early work used high power laser beams focused perpendicular to the electrode axis. This configuration triggered the gap by creating a localized field distortion in the gap without a laser created spark. ${ }^{1}$ Improved performance was obtained by focusing lower power lasers onto one of the electrodes of the spark gap to create a plasma that initiates the closing of the switch. In this scheme, the electric field amplifies the laser created plasma ionization until a conductive path is created via an electron avalanche and streamer formation, closing the switch. ${ }^{2}$ This method used primarily ruby, $\mathrm{CO}_{2}$, and Nd:YAG lasers with pulse energies ranging from several Joules ${ }^{3,4}$ to $0.17 \mathrm{~mJ},{ }^{5,6}$ and is well reviewed by Guenther and Bettis, ${ }^{2}$ and re-examined by Dougal and Williams. ${ }^{7}$ Using this configuration, subnanosecond jitter can be obtained when the spark gap is operated close to the self-breakdown voltage. ${ }^{2}$ The latest developments have involved creating an ionized channel in the gas using UV light from $\mathrm{KrF}^{8,9}$ or quadrupled $\mathrm{Nd}$ : $\mathrm{YAG}^{10}$ lasers. These volume interaction techniques have allowed fast switching with subnanosecond jitter down to $80 \%$ of the selfbreakdown voltage. Recently, a significant amount of research has also been reported in the related study of self-guiding ${ }^{11-15}$ and laser discharging of lightning ${ }^{16,17}$ by femtosecond pulses.

The widespread availability of femtosecond Ti:Sapphire amplifiers with output pulse energies of a few $\mathrm{mJ}$ offers the opportunity to easily exceed the intensities necessary to tunnel-ionize atoms and molecules, ${ }^{18}$ and at the same time, the application of these lasers creates a need for their accurate synchronization with events controlled by high-voltage spark gaps. Herein, we report sub-100 ps jitter operation of a pressurized high-voltage spark gap triggered by femtosecond Ti:Sapphire laser pulses with an energy of a fraction of a mJ.

The experimental setup is shown in Fig. 1. The laser pulses of a KM Labs laser oscillator were stretched to about 200 ps using a grating stretcher and were amplified to $1-2$

${ }^{\text {a)} E l e c t r o n i c ~ m a i l: ~ b m l @ l a m a r . c o l o s t a t e . e d u ~}$
$\mathrm{mJ}$ in eight passes through a Ti:Sapphire $\operatorname{rod}^{19}$ pumped by 20 $\mathrm{mJ}$ pulses from a frequency doubled Nd:YAG laser. The pulses were recompressed to about 50 fs using a grating compressor. The pressurized spark gap consisted of a pair of brass or molybdenum electrodes separated by distances ranging from 0.1 to $0.3 \mathrm{~cm}$. In all the experiments reported herein, the spark gap was pressurized with dry air. The laser beam was introduced into the spark gap through an axial orifice. The spark gap was tested at a repetition rate of $10 \mathrm{~Hz}$ discharging a $1.7 \mathrm{nF}$ high-voltage capacitor though a low inductance circuit. The time delay jitter between the arrival of the laser pulse and the peak of the current pulse was
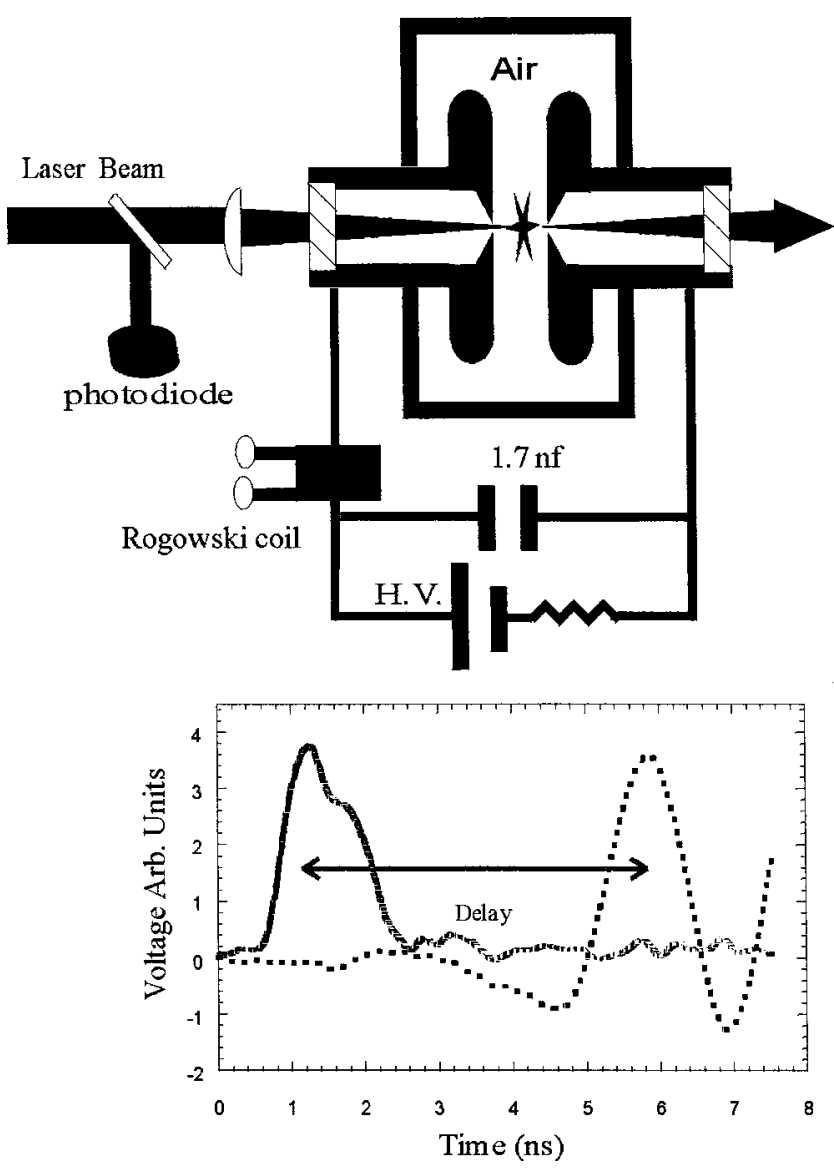

FIG. 1. Schematic representation of the experimental setup. The laser beam was focused into the spark gap by an $f=10 \mathrm{~cm}$ lens. Typical laser (solid line) and current pulses (dashed line) are shown. 


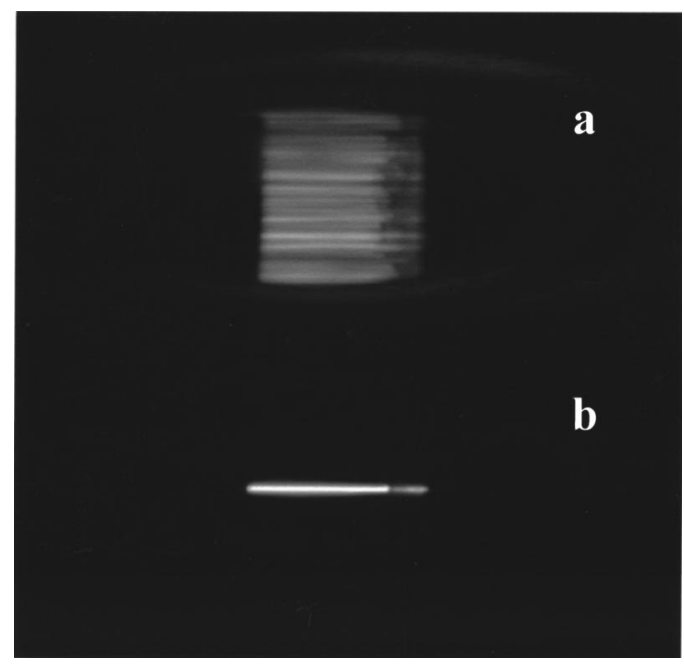

FIG. 2. (a) Open shutter photograph of free-firing spark gap. Discharge channels developed over multiple shots can be seen. (b) Open shutter photograph of laser-triggered spark gap over multiple shots.

measured using an 8 Gigasample/s digitizing oscilloscope. Typical current and laser pulse traces are shown in Fig. 1. The delay between the laser and current pulses was defined as the time interval between their maxima. The jitter is reported as the standard deviation of the delay time (jitter $=1 \sigma$ ). The spark gap was generally run at $\sim 90 \%$ of the self-breakdown voltage, but lower voltages were also studied. Figure 2 shows photographs of the random channel formation characteristic of the self-triggered (over voltage) mode of operation [Fig. 2(a)], as opposed to the reproducible guided discharge channel produced by the laser trigger [Fig. 2(b)]. The effect of the laser pulse energy on jitter and delay are shown in Fig. 3. In this configuration, the laser pulse propagates axially through holes drilled in both the cathode and the anode, and the laser is focused in the midgap creating a visible spark. The results shown in Fig. 3 are for brass electrodes separated by $2.3 \mathrm{~mm}$ discharging $10 \mathrm{kV}$ at an absolute pressure of 22 psi. Figure 3 shows that the spark gap can be run at subnanosecond jitter with pulse energies as low as $0.1 \mathrm{~mJ}$. The associated delays are also shown. Both the time delay and jitter show a nearly exponential increase with decreasing laser power. From Fig. 3, it can be seen that

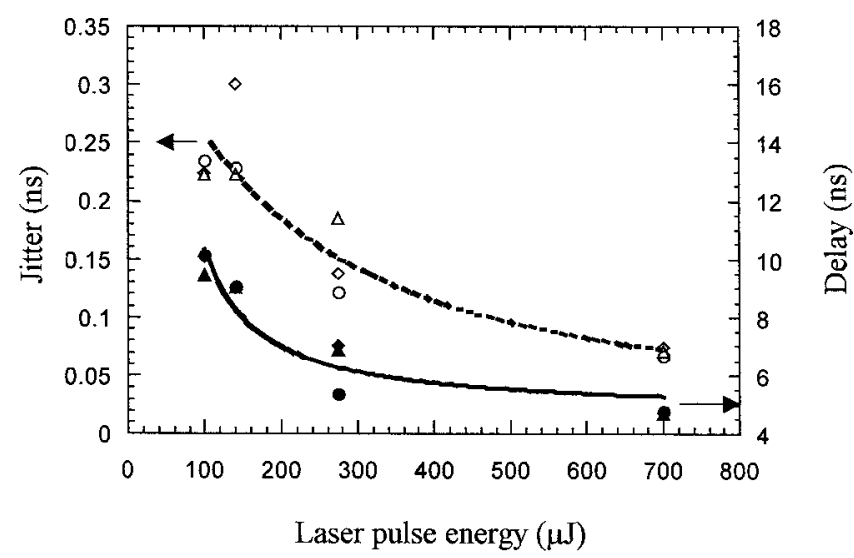

FIG. 3. (a) Spark gap jitter vs laser pulse energy obtained focusing the laser on the electrode midgap. The electrode separation was $2.3 \mathrm{~mm}$ at an absolute dry air pressure of $22 \mathrm{psi}$. The voltage was $10 \mathrm{kV}$. Symbols represent individual 300 shot runs. (b) Delays associated with jitters in (a).

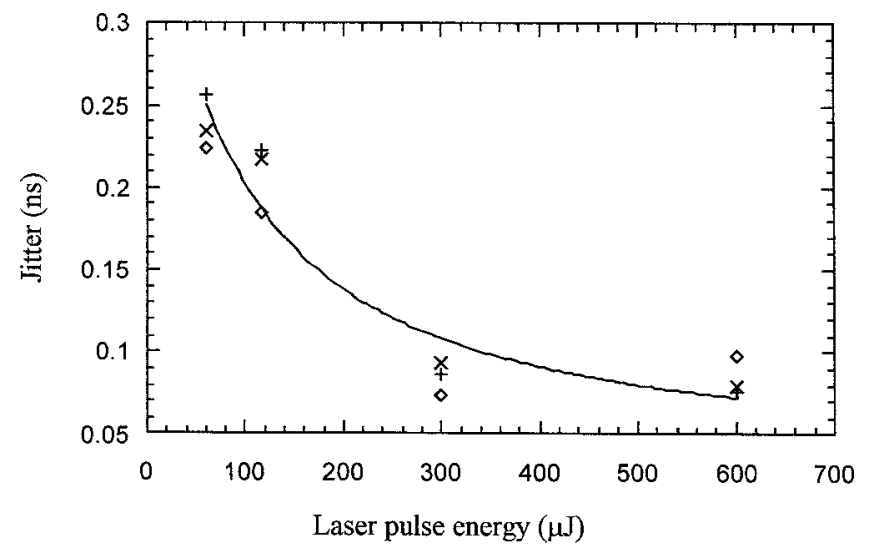

FIG. 4. (a) Spark gap jitter vs laser pulse energy obtained by focusing the laser beam on a brass anode. Electrode separation was $1.26 \mathrm{~mm}$ at an absolute dry air pressure of $39 \mathrm{psi}$. The voltage was $10 \mathrm{kV}$. Symbols represent individual 300 shot runs.

high-voltage spark gaps can be triggered effectively using only a small portion of the energy typically available from single stage amplified Ti:Sapphire systems (multipass or regenerative amplifiers). We have also investigated a second configuration in which the laser pulse passes through a hole in the first electrode and is then focused on the second electrode. Jitter measurements for this configuration are shown in Fig. 4 for a spark gap with brass electrodes separated by 1.26 $\mathrm{mm}$, operated at an absolute pressure of 39 psi. For this experiment, the laser was focused on the anode and the initial voltage across the gap was $10 \mathrm{kV}$. In this configuration, pitting of the electrode was observed but did not effect the jitter over thousands of shots. Comparison of Figs. 3 and 4 reveals that the spark gap can be operated in either configuration with $\sigma \sim 100 \mathrm{ps}$ jitter. The fact that the ablation-mode data in Fig. 4 shows lower jitter is likely due to the smaller gap distance.

Figure 5 shows the dependence of jitter on the percentage of the self-breakdown voltage for a $1.26 \mathrm{~mm}$ gap with a self-breakdown voltage of $11 \mathrm{kV}$ triggered in the ablation configuration. As expected, the jitter is seen to improve as the percentage of the self-breakdown voltage increases, while subnanosecond jitter is accomplished at voltages less than $65 \%$. Figure 6 shows the delay distribution for an optimized 900 shot run discharging $10 \mathrm{kV}$, in which the triggering configuration was an ablation of a Mo cathode separated

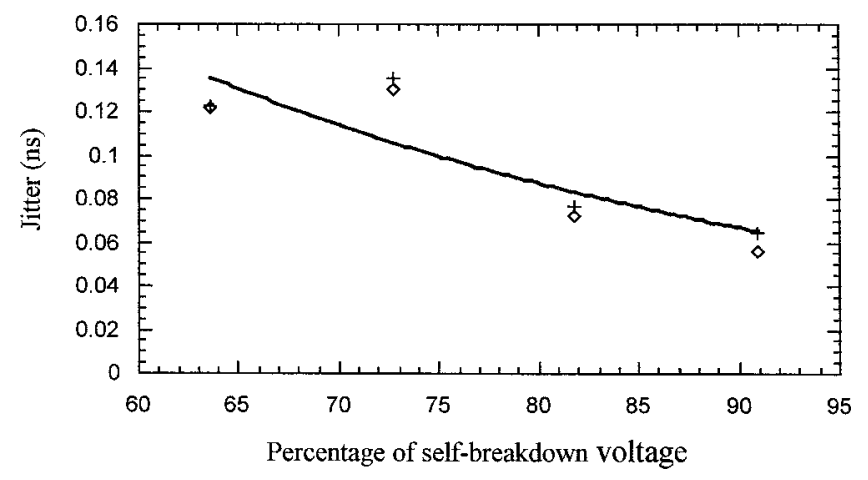

FIG. 5. Jitter as a function of self-breakdown voltage. The spark gap was triggered by ablation of the anode electrode. The electrode separation was $1.26 \mathrm{~mm}$ at an absolute dry air pressure of $39 \mathrm{psi}$. The voltage was $11 \mathrm{kV}$. 


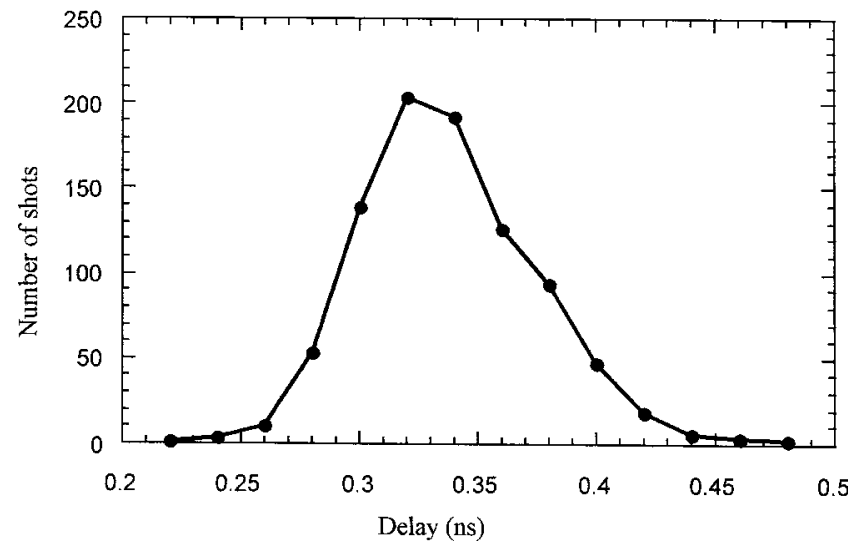

FIG. 6. Distribution of time delays for an optimized 900 shot run with a molybdenum cathode. The spark gap was triggered by ablation of the cathode. Electrode separation was $1.0 \mathrm{~mm}$ and the absolute dry air pressure was $26.5 \mathrm{psi}$. The voltage was $10 \mathrm{kV}$. The standard deviation is $\sigma=0.037 \mathrm{~ns}$.

from a brass anode by $1 \mathrm{~mm}$. The distributions shows a jitter of $+-\sigma=0.035 \mathrm{~ns}$.

In the experiments discussed herein, free electrons are created by optical field induced ionization in the intense field of the ultrashort pulse laser, as well as by ablation. Computation of the Keldysh parameter ${ }^{18} \quad \gamma=\omega\left(2 m_{e} E_{i p}\right)^{1 / 2} / e E$ (where $\omega$ is the angular frequency of the light, $E$ is the laser electric field, and $E_{i p}$ is the ionization potential of gas) for the ionization of oxygen molecules at our experimental condition of $\lambda_{\text {laser }}=800 \mathrm{~nm}, 50 \mathrm{fs}$ laser pulse width, and pulse energies of 0.6 and $0.1 \mathrm{~mJ}$ focused to $20 \mu \mathrm{m}$ diameter spot, yield values of $\gamma=0.16$ and 0.4 , respectively. These values of $\gamma<1$ indicate that the laser intensities used here exceed the value necessary for tunnel ionization. The similarity in the results for focusing on the electrode and the gap (Figs. 3 and 4) suggests that in both cases sufficient ionization might occur in the gas to rapidly close the switch.

In summary, we have focused Ti:Sapphire femtosecond laser pulses of a fraction of $\mathrm{mJ}$ energy to intensities exceed- ing the values necessary for tunnel ionization of air to demonstrate sub-100 ps jitter operation of high-voltage spark gaps. The results will allow for accurate synchronization between a femtosecond laser and high current pulses in a significant number of applications.

The authors gratefully acknowledge the support from the U.S. Department of Energy Office of Basic Energy Sciences, and the National Science Foundation. The authors also acknowledge the generous support from the W. M. Keck Foundation, and thank S. Backus, M. Murnane, and H. Kapteyn for their assistance with the multipass amplifier.

${ }^{1}$ W. K. Pendelton and A. H. Guenther, Rev. Sci. Instrum. 36, 1546 (1965).

${ }^{2}$ A. H. Guenther and J. R. Bettis, J. Phys. D 11, 1577 (1978).

${ }^{3}$ A. H. Guenther and J. R. Bettis, IEEE J. Quantum Electron. 3, 581 (1967).

${ }^{4}$ A. V. Nurmiko, IEEE J. Quantum Electron. 7, 470 (1971).

${ }^{5}$ J. R. Bettis and A. H. Guenther, IEEE J. Quantum Electron. 6, 483 (1970).

${ }^{6}$ F. Pinnekamp, G. Himmel, and K. Bergstedt, Opt. Commun. 11, 225 (1974).

${ }^{7}$ R. A. Dougal and P. F. Williams, J. Phys. D 17, 903 (1984).

${ }^{8}$ M. J. Kushner, R. D. Milroy, and W. D. Kimura, J. Appl. Phys. 58, 2988 (1985).

${ }^{9}$ W. D. Kimura, M. J. Kushner, and J. F. Seamans, J. Appl. Phys. 63, 1882 (1988).

${ }^{10}$ J. R. Woodworth, P. J. Hargis, L. C. Pitchford, and R. A. Hamil, J. Appl. Phys. 56, 1382 (1984).

${ }^{11}$ S. Tzortzakis, M. A. Franco, Y.-B. Andre, A. Chiron, B. Lamouroux, B. S. Prade, and A. Mysyrowicz, Phys. Rev. E 60, R3505 (1999).

${ }^{12}$ A. Talebpour, S. Petit, and S. L. Chin, Opt. Commun. 171, 285 (1999).

${ }^{13}$ H. D. Ladouceur, A. P. Baronavski, D. Lohrmann, P. W. Grounds, and P. G. Girardi, Opt. Commun. 189, 107 (2001).

${ }^{14}$ M. Mlejnek, E. M. Wright, and J. Moloney, IEEE J. Quantum Electron. 35, 1771 (1999).

${ }^{15}$ S. Petit, A. Talebpour, A. Proulx, and S. L. Chin, Opt. Commun. 175, 323 (2000).

${ }^{16}$ B. La Fontaine, F. Vidal, D. Comtois, C. Y. Chien, A. Desparious, T. W. Johnston, J. C. Kieffer, H. P. Mercure, H. Pepin, and F. A. M. Rizk, IEEE Trans. Plasma Sci. 27, 688 (1999).

${ }^{17}$ H. Shillinger and R. Sauerbrey, Appl. Phys. B: Lasers Opt. 68, 753 (1999).

${ }^{18}$ L. V. Keldysh, Sov. Phys. JETP 20, 1307 (1965).

${ }^{19}$ S. Backus, C. G. Durfee III, G. Mourou, H. C. Kapteyn, and M. M. Murnane, Opt. Lett. 22, 1256 (1997). 